\title{
Gas phase Raman spectroscopy : comparison of continuous wave and cavity based methods
}

\author{
Lee Weller ${ }^{1}$, Maxim Kuvshinov ${ }^{1}$ and Simone Hochgreb ${ }^{1}$ \\ ${ }^{1}$ Advanced Nanotube Application and Manufacturing Initiative (ANAM), Department of Engineering, University of \\ Cambridge, Trumpington Street, Cambridge, CB2 1PZ, UK \\ January 24, 2018 \\ lw493@cam.ac.uk
}

\begin{abstract}
Comparison of cavity-enhanced Raman spectroscopy to continuous wave detection for gas phase molecules in air. We show continuous measurements with calculated emission and discuss the potential benefits (two orders more signal) of using a cavity. @ 2018 The Author(s)
\end{abstract}

OCIS codes: 140.3550 - Lasers, Raman, 170.5660 - Raman spectroscopy, 190.5650 - Raman effect

Spontaneous Raman scattering is a valuable diagnostic tool that has found use in many different fields of research and industry. In chemical kinetics, vibrational spectroscopy has found use for Raman flame measurements in combustion and Thomson scattering measurements of low-density plasmas. In addition to this, the chemical pathway for many reacting flows has much interest for simulations, design and automation. Raman allows the ability to follow simple gas species such as oxygen, nitrogen and carbon dioxide during process control providing a powerful method for quantitative gas phase analysis and trace gas detection for environmental monitoring. Safety monitoring of hydrogen gas levels, isotope tracer studies and monitoring radioactive tritium is paramount not only to atmospheric sensing research however to the health and safety of many work places in industry. Surface chemistry and catalysis include refining the theoretical analysis of molecular energy levels and potential energy hypersurfaces. Furthermore, due to its high-resolution, there is potential for increased fundamental scientific understanding of vibrational interactions and intramolecular vibrational energy redistribution on faster timescales. These different fields tend to use high power laser (CW) systems with large footprints. Here we propose a technique called Cavity Enhanced Raman Spectroscopy (CERS). Including the already increasing range of applications, this technique would also motivate real time, low cost, small footprint in situ measurements in a broad range of other scientific and industrial fields from NEMs/MEMs systems, organic electronics and Nano/Microscale chemistry to planetary atmospheric detection, exo-meteorology, deep-sea explorations [1], human breath analysis [2], turbine power plant [3] and fermentation gases [4]. Real time analysis capabilities, and continuous Raman signals with low power diodes, makes the cavity technique none invasive and applicable for future applications.

A detailed calculation of the rate of emission of Raman photons in such systems can be found in [5]. The rate of emission per molecule in free space scaled by the input power of the laser is given by

$$
\frac{\gamma_{S}}{P_{\mathrm{in}} N}=R_{f} \frac{\lambda_{S}^{4}}{\Delta \lambda_{S}^{2}} \frac{\mathrm{d} \sigma}{\mathrm{d} \Omega}
$$

where $R_{f}=3 d_{0}^{3} / 64 h c f^{3}$ is a constant based on the optical arrangement, $\mathrm{d} \sigma / \mathrm{d} \Omega$ is the differential Raman scattering cross section, with $\lambda_{S}$ and $\Delta \lambda_{S}$ being the Stokes wavelength and linewidth, respectively. For a typical setup one can expect the magnitude of $R_{f}$ to be $\approx 5 \times 10^{16}$. The maximum amount of photons are expected for a long wavelength laser with high power over a narrow linewidth. Note $\mathrm{d} \sigma / \mathrm{d} \Omega$ also has a wavelength dependence. There are three main factors that contribute to the enhancement of spontaneous Raman scattering within a cavity: (1) Resonant recirculation describes the power build up within the cavity, (2) Purcell effect describes the increase in the rate of spontaneous emission due to the presence of a cavity, (3) Double resonance condition describes that the cavity length must be set such that the number of free spectral ranges equals the shift of the Raman mode of interest. The rate of emission per molecule in a cavity scaled by the input power of the laser is given by

$$
\frac{\gamma_{s}}{P_{\mathrm{in}} N}=R_{c} \frac{\lambda_{S}^{6}}{\Delta \lambda_{p}^{(c)} \Delta \lambda_{S}} \frac{\mathrm{d} \sigma}{\mathrm{d} \Omega},
$$




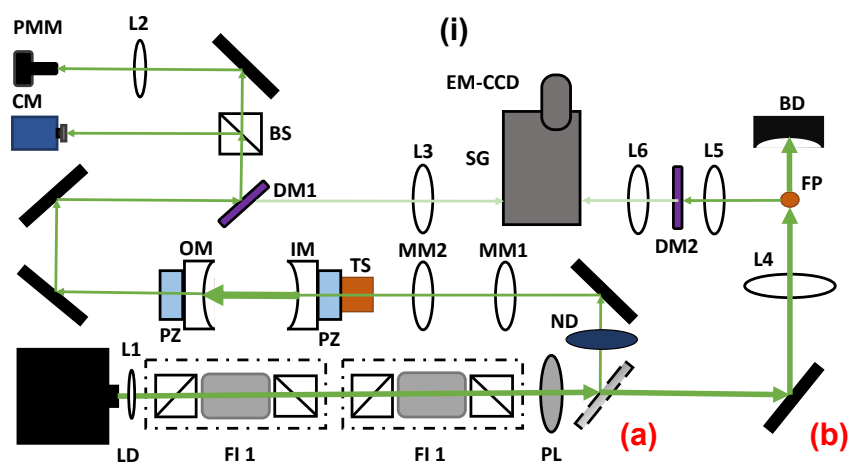

(ii)

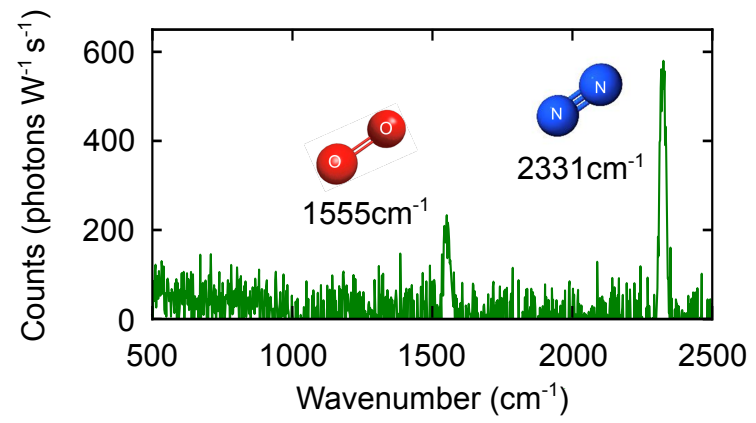

Fig. 1. (i) Experimental setup for gas phase Raman detection with (a) CERS and (b) CW. (ii) Gas phase Raman detection of room air using CW.

where $R_{c}=2 \tan ^{-1}(l / \sqrt{l(2 R O C-l)}) / h c \pi^{2} l^{2}$ is a constant based on the optical arrangement, $l$ being the length of the cavity, $R O C$ being the radius of curvature of the mirrors and $\Delta \lambda_{p}^{(c)}$ being the pump linewidth within the cavity. For a typical setup one can expect the magnitude of $R_{c}$ to be $\approx 4 \times 10^{27}$. The maximum amount of photons are expected for a cavity length of a few mm, a small ROC $(R O C>l / 2)$ and a small $\Delta \lambda_{p}^{(c)}$ defined by the Finesse of the cavity. The ratio of rates of emission for a molecule in a cavity compared to free space is equal to

$$
\text { ratio }=\frac{R_{c}}{R_{f}} \frac{\Delta \lambda_{S}}{\Delta \lambda_{p}^{(c)}} \lambda_{S}^{2} \approx \frac{0.001 \mathrm{~nm}}{\Delta \lambda_{p}^{(c)}} .
$$

For a molecule in a cavity with finesse on the order of $6 \times 10^{4}$, one can expect two orders of magnitude more signal (for the same input power) in comparison to one in free space. Hence one would need a laser with two orders of magnitude more power to achieve the same amount of signal. Note the FSR for a cavity of length a few $\mathrm{mm}$ is approximately tens of $\mathrm{GHz}$, hence for a cavity illuminated in the visible there are several hundred enhanced scattered modes approximately every one tenth of a nm.

Fig. 1 (i) show the experimental setups used to carry out (a) CERS and (b) CW measurements, respectively. CERS measurements couple light into a linear optical cavity consisting of two highly reflecting mirrors. CW measurements adopt a $90^{\circ}$ lens collection setup. Both measurements use dichroic filters to separate out the intense Rayleigh photons from the Stokes photons before being collected using a spectrograph coupled with an EM-CCD camera. Fig. 1 (ii) shows measured Raman lines in room air for $\mathrm{O}_{2}\left(1555 \mathrm{~cm}^{-1}\right)$ and $\mathrm{N}_{2}\left(2331 \mathrm{~cm}^{-1}\right)$. Eq. 1 predicts a normalised signal for $\mathrm{N}_{2}$ in air of 1400 photons $\mathrm{W}^{-1} \mathrm{~s}^{-1}$ for a laser power of $1 \mathrm{~W}$. In fig. 1 (ii) we measure approximately 600 photons $\mathrm{W}^{-1} \mathrm{~s}^{-1}$ which is less than the predicted value. The reason for this is that eq. 1 assumes that both the collection and illuminated volumes are perfectly overlapped and of the same size. In addition there are losses as not all the Stokes photons are transmitted through the collection optics. In conclusion we have shown gas phase Raman spectroscopy measurements using a CW system and compared the potential benefits (several orders of magnitude more signal) for a cavity system with a relatively high Finesse. Note there are many papers that use fibres to enhance Raman signatures, however the aim of this work is to measure molecules in-situ.

\section{References}

1. D. Yang, J. Guo, Q. Liu, Z. Luo, J. Yan and R. Zheng, "Highly sensitive Raman system for dissolved gas analysis in water," Appl. Optics. 55 7744-7748 (2016).

2. K. K. Chow, M. Short, S. Lam, A. McWilliams and H. Zeng, "A Raman cell based on hollow core photonic crystal fiber for human breath analysis," Med. Phys. 41092701 (2014).

3. J. Kiefer, T. Seeger, S. Steuer, S. Schorsch, M. C. Weikl and A. Leipertz, "Design and characterization of a Raman-scattering-based sensor system for temporally resolved gas analysis and its application in a gas turbine power plant,” Meas. Sci. Technol. 19085408 (2008).

4. Y. Numata, Y. Shinohara, T. Kitayama and H. Tanaka "Rapid and accurate quantitative analysis of fermentation gases by Raman spectroscopy," Process. Biochem. 48 569-574 (2013).

5. B. J. Petrak, "Microcavity Enhanced Raman Scatttering," Thesis (2016). 